\title{
Production Optimization Based on Integrated Modeling and Gradient Methods
}

\author{
Meysam Naderi ${ }^{1} \mathbb{D}$, Ehsan Khamehchi ${ }^{1, *} \mathbb{D}$, Behrooz Karimi ${ }^{2,}$ \\ 1 Department of Petroleum Engineering, Amirkabir University of Technology, Tehran, Iran, \\ 2 Department of Industrial Engineering and Management Systems, Amirkabir University of Technology, Tehran, Iran \\ * Correspondence: Khamehchi@aut.ac.ir;
}

Scopus Author ID 26657370700

Received: 8.06.2020; Revised: 1.07.2020; Accepted: 1.07.2020; Published: 3.07.2020

\begin{abstract}
Hydrocarbon production from a large-scale field requires optimal strategies in order to sustain returns. However, the determination of optimal plans for production is a challenging task due to high computational cost and various nonlinear constraints. Therefore, an efficient methodology is required to determine the optimum criteria with minimum cost and maximum precision. In this paper, two quasi-newton second-order algorithms of the Broyden-Fletcher-Goldfarb-Shanno (BFGS) and symmetric rank one (SR1) are used to maximize the net-present-value (NPV) over 17 years of production and injection. The framework is applied to an integrated simulation model of reservoir, wells, and surface facilities of a real oil field located in the Middle East. The Lagrangian method is used to combine the objective function and constraints during optimization. The results obtained are compared with those of the current production scheduled plan provided by the oil industry. The performance of algorithms is affected by the production optimization time scale. For the first 12 years of production optimization, SR1 outperform BFGS. For the last 5 years, BFGS outperforms SR1. However, for all years of production, BFGS is superior over SR1 and increases NPV over the base case NPV from $1.605 \%$ to $4.92 \%$ for various time steps of production.
\end{abstract}

Keywords: Integrated Field production optimization; quasi-newton; BFGS; SR1.

(C) 2020 by the authors. This article is an open-access article distributed under the terms and conditions of the Creative Commons Attribution (CC BY) license (https://creativecommons.org/licenses/by/4.0/).

\section{Introduction}

Production from mature oil fields could be suboptimal due to their suboptimal development and production plans over the past years. In order to maintain the returns, it is required to optimize the future and current scheduled production and injection strategies. The optimization process should be performed to maximize the ultimate oil recovery with minimized costs. However, the production optimization of a mature hydrocarbon field is computationally expensive due to its time-consuming nature and a large number of decision variables. In addition, the complexity of the optimization increases by the existence of operational constraints, the nonconvex nature of the problem, and the formulation of the problem. Therefore, it is crucial to propose an efficient and suitable framework for large-scale oil field production optimization in such a way to result in maximum profit with a minimum number of function evaluations.

Several authors have applied various algorithms for production optimization including genetic algorithm combined with an artificial neural network for nonconventional wells optimization [1], well placement using hybridized genetic algorithm and gradient-based method [2,3], placement of vertical wells using semi analytic method [4], field-development 
using particle-swarm-optimization [5], retrospective optimization and covariance matrix adaptation evolution strategy for optimizing well locations and trajectories [6, 7], optimal well number and type, sequence of drilling and their corresponding locations and controls using particle swarm optimization and mesh adaptive direct search [8], genetic algorithm for longterm gas-lift allocation optimization [9], nonlinear risk approach based on genetic algorithm for production optimization [10], reduced order modelling coupled with a guided pattern search algorithm [11], metaheuristic bat algorithm for well placement optimization [12], bat algorithm combined with design of experiment techniques for well placement and individual well controls optimization [13], and stochastic simplex approximate gradient [14-16], design of experiment [17-19], convolutional neural network based proxy [20], multipoint adaptive gaussian process surrogate model with important design domain [21], augmented lagrangian and sequential quadratic programming algorithms [22], cooperative micro-particle swarm optimization [23], fuzzy c-mean clustering algorithm and loop optimization method [24], reduced sampling strategy coupled with simultaneous perturbation stochastic approximation algorithm [25], self-adapt reservoir clusterization method with artificial kohonen neural network [26], well control zonation and mixed integer nonlinear programming [27], deep neural network models [28], long short-term memory neural network based model coupled with particle swarm optimization algorithm [29], robust optimization [30], just to name a few

A review of previous studies shows that generally, two types of gradient-based and derivative-free algorithms have been used for production optimization. Because of the timeconsuming nature of reservoir simulation and optimization process, most of the previous reservoir models used are based on synthetic or semi-synthetic models, and without considering surface facilities. In other words, few research efforts have focused on mature oil field optimization to validate the optimization results and also by considering surface facilities constraints. Therefore, in the current study, the reservoir model is a real large scale, and it is coupled by a surface facility during optimization. Since gradient-based and gradient-free optimization algorithms have advantages and disadvantages, the proper selection is problemdependent. Based on the problem nature, the number of decision variables, and the degree of convergence, the typical gradient-free algorithm requires from 5 to 200 times as many function evaluations as the gradient-based algorithm. Therefore, the gradient information can significantly improve the speed of convergence relative to a method that does not compute gradients. However, the gradient-based algorithms find a local rather than a global optimum. This is unlikely to be an issue as a result of a highly constrained problem and solution strategies such as convex programming. In the current production optimization, applying meta-heuristic algorithms for optimization of large-scale fields is computationally prohibitive due to a large number of parameters and function evaluations prior to reaching an acceptable solution. Therefore, it is required to select the most appropriate optimization algorithms in order to be able to reach a trade-off between computational cost and the quality of obtained solutions for the problem under consideration. In this regard, two quasi-newton methods based on the Broyden-Fletcher-Goldfarb-Shanno (BFGS) algorithm, and symmetric rank one (SR1) are applied to an integrated simulation model of reservoir, wells and surface facilities for production optimization. The objective function is the net present value of 17 years of production, which should be maximized by adjusting individual good production and injection rates on specified control steps.

This paper is organized as follows. Section 2 gives an explanation of the real oil field with its geological and fluid properties. Section 3 describes the formulation of the production 
optimization problem, the calculation of the objective function gradient, and the quasi-newton second-order gradient-based algorithms of BFGS and SR1 to maximize NPV. Section 4 reviews and discusses the results of the proposed optimization framework. Finally, the main findings are presented in Section 5.

\section{Materials and Methods}

\subsection{Model description.}

In order to perform the required reservoir simulations for production optimization, a real, full-scale, mature oil field located in the southwest of Iran was selected. The simulation model is an integrated model of a hydrocarbon reservoir, wells, and surface facilities, including production and injection manifolds, horizontal pipelines, separators, and compressors for gas injection. The model was characterized as a large-size industrial reservoir engineering model. Corner-point geometry has been used to model the geometry of the field. The integrated model contains a three-phase gas-oil-water system with $83 \times 115 \times 28$ grid blocks, of which 156631 blocks are active. The field contains 4 gas injectors and 27 producers that have been completed in the oil column. The number of active production wells is 19 , and the number of active injection wells is 3 . All wells are vertical, and the wells operate under constant-rate production constraints. After falling below a limiting bottom hole pressure (BHP), they will switch to a BHP-constraint.

Table 1. Engineering and general reservoir data.

\begin{tabular}{l|l} 
Properties & Value \\
\hline Producing tubing inside diameter & $2.996 \mathrm{in.}$ \\
\hline Top of producing sand face & $13466 \mathrm{ft}$. \\
\hline Wellhead temperature & $77 \mathrm{~F}$ \\
\hline Production fluid & Oil \\
\hline Thickness of producing layer & $100-300 \mathrm{ft}$. \\
\hline Wellbore radius & $0.36 \mathrm{ft}$. \\
\hline Reservoir pressure & $8800 \mathrm{psi}$ \\
\hline Reservoir temperature & $294.3 \mathrm{~F}$ \\
\hline
\end{tabular}

Figure 1. 3D reservoir model of the oil field with the location of all production and injection wells (green and blue colors represent oil and water, respectively).

The minimum bottom hole pressure is $100 \mathrm{psi}$. The reservoir has been developed under gas injection for 18 years. The injected gas is associate gas. Figure 1 shows the simulated 3D model of this oil field with well locations in the reservoir. Schlumberger Eclipse 300 software is used for performing reservoir simulations. The production optimization is to find good optimal controls (injection rates for injectors and production rates for producers) for a production period of 17 years of reservoir life. The length of each control step is equal to 365 
days. The simulation run time for 17 years of production is 165 minutes using a PC with CPU of $2.5 \mathrm{GHz}$ and $32 \mathrm{~GB}$ of memory RAM. The good control variables at each injector are the injection rates with an upper bound of $105 \mathrm{MMscf} / \mathrm{D}$ and a lower bound of zero, and each producer operates under oil rate control with a specified upper bound of 1800 psi for tubing head pressure (THP). The maximum field injection rate is $280 \mathrm{MMscf} / \mathrm{D}$ for three active gas injection wells. The reservoir is currently under-saturated and therefore only oil and water flow in the reservoir. The engineering and general reservoir data, geological and fluid properties of the reservoir are provided in Table 1-2. The three-phase relative permeability data is given in Table 3.

Table 2. Fluid and rock properties.

\begin{tabular}{l|l} 
Properties & Value \\
\hline Solution gas oil ratio & $1.721 \mathrm{MSCF} / \mathrm{STB}$ \\
\hline Oil gravity & $33.71 \mathrm{API}$ \\
\hline Gas gravity & 0.83 sp. gravity \\
\hline Oil viscosity & 0.296 centipoise \\
\hline Oil formation volume factor & $1.898 \mathrm{RB} / \mathrm{STB}$ \\
\hline Bubble point pressure & $4887 \mathrm{Psi}$ \\
\hline Skin factor & $0-25$ \\
\hline Average reservoir permeability & $110 \mathrm{md}$ \\
\hline Average reservoir temperature & $260 \mathrm{~F}$
\end{tabular}

Table 3. Three-phase relative permeability data.

\begin{tabular}{l|l|l} 
So & Krow & Krog \\
\hline 0.00 & 0.00 & 0.00 \\
\hline 0.08 & 0.00 & 0.00 \\
\hline 0.13 & 0.00 & 0.00 \\
\hline 0.18 & 0.00 & 0.00 \\
\hline 0.23 & 0.00 & 0.00 \\
\hline 0.28 & 0.00 & 0.00 \\
\hline 0.33 & 0.00 & 0.02 \\
\hline 0.35 & 0.00 & 0.03 \\
\hline 0.38 & 0.00 & 0.05 \\
\hline 0.40 & 0.00 & 0.08 \\
\hline 0.43 & 0.01 & 0.10 \\
\hline 0.46 & 0.02 & 0.13 \\
\hline 0.48 & 0.03 & 0.16 \\
\hline 0.51 & 0.04 & 0.19 \\
\hline 0.53 & 0.06 & 0.22 \\
\hline 0.56 & 0.08 & 0.26 \\
\hline 0.58 & 0.10 & 0.29 \\
\hline 0.59 & 0.11 & 0.30 \\
\hline 0.61 & 0.14 & 0.34 \\
\hline 0.63 & 0.16 & 0.37 \\
\hline 0.64 & 0.17 & 0.38 \\
\hline 0.67 & 0.21 & 0.43 \\
\hline 0.68 & 0.24 & 0.46 \\
\hline 0.69 & 0.25 & 0.48 \\
\hline 0.72 & 0.30 & 0.53 \\
\hline 0.73 & 0.33 & 0.55 \\
\hline 0.74 & 0.36 & 0.58 \\
\hline 0.77 & 0.41 & 0.63 \\
\hline 0.78 & 0.44 & 0.65 \\
\hline 0.80 & 0.48 & 0.69 \\
\hline 0.82 & 0.55 & 0.75 \\
\hline 0.84 & 0.60 & 0.80 \\
\hline 0.85 & 0.62 & 0.84 \\
\hline 0.88 & 1.00 & 1.00 \\
\hline & & \\
\hline 0.53 \\
\hline 0.53
\end{tabular}




\section{Results and Discussion}

\subsection{Methodology.}

Two types of gradient-based algorithms and derivative-free algorithms have been investigated to solve various production optimization problems in the petroleum industry. In gradient-based algorithms, the gradient information of the objective function is used in searching for optimal solutions, while the derivative-free algorithms do not rely on derivative information to find optimal solutions. Derivative-free algorithms evaluate the objective function many times in the domain of variables to reach the optimal solution. The steepest ascent method, the simultaneous perturbation stochastic approximation algorithm, and conjugate gradient methods are typical gradient-based methods. The typical derivative-free algorithms could be a genetic algorithm, particle swarm optimization, and meta-heuristic bat algorithm. The optimization algorithm should be selected based on the nature of the problem being solved, a number of optimization variables, and the required time for reservoir simulation. For the large-scale full field production optimization in the current study, it is computationally prohibitive to use derivative-free algorithms. In this regard, the second-order gradient-based algorithms are applied as one of the earliest works for the integrated production optimization of a real mature oil field. The following subsections give a detailed description of the proposed optimization framework.

\subsubsection{Problem formulation.}

The generalized optimization problem for mature fields includes individual good rate controls (continuous variables) and well placement (integer variables). However, for the current study, only well rates are going to be optimized. The number of new wells and the corresponding well locations could also be investigated. The most common objective function for production optimization based on literature is the net-present-value (NPV) of production, which can be calculated as follow:

$$
N P V(u)=\sum_{n=1}^{N T S} \frac{P_{o} Q_{o}+P_{g} Q_{g}-C_{w} Q_{w}-C_{i n j g} Q_{i n j g}-C_{i n j w} Q_{i n j w}-O P E X}{(1+i)^{n}}-C A P E X
$$

where $u$ is a vector of all good controls over the production lifetime; $n$ denotes the nth time step of the reservoir simulator; NTS is the total number of time steps; $i$ is the annual discount rate; $P_{o}(\mathrm{USD} / \mathrm{STB})$ is the sales price of oil; $P_{g}(\mathrm{USD} / 1000 \mathrm{SCF})$ is the sales price of gas; $C_{w}$ and $C_{i n j w}$, respectively, denote the disposal cost of produced water and the cost of water injection, in units of USD/STB; $C_{\text {injg }}$ is the cost of gas injection (USD/1000 SCF), $Q_{o}$ is the cumulative oil production (STB); $Q_{g}$ is the cumulative gas production (SCF); $Q_{w}$ is the cumulative water production (STB); $Q_{i n j g}$ is the cumulative gas injection (SCF); $Q_{i n j w}$ is the cumulative water injection (STB); OPEX is the operational expenditure (USD); CAPEX is the capital expenditure (USD), and $N P V$ is the net present value (USD).

The generalized production optimization problem for mature fields can be stated as follows: $\max J(u)$

$$
\text { s.t. } g(u)=0 \text {, and } c(u) \leq 0
$$

where $J$ is the objective function, either the net present value (NPV) or the cumulative oil produced; $g$ and $c$ are equality and inequality constraints, respectively; and $u$ denotes the good production variables, which are either bottom hole pressures or injection/production rates. This 
constrained optimization is solved using the Lagrangian method to combine the objective function of NPV and various linear and nonlinear constraints as an unconstrained optimization problem. The Lagrangian form of the Eq. 2 is as follow:

$$
\max L=J(u)-\lambda g(u)-\mu c(u)
$$

where $L$ is Lagrangian, $\lambda$ and $\mu$ denote Lagrangian multiplier. The optimum values of all controls and Lagrangian multipliers are determined using quasi-Newton methods, as explained in detail in Section 3.2. The individual well controls and surface facility constraints are maximum oil production rate for producers, maximum gas injection rate for injectors, minimum bottom hole pressure of $100 \mathrm{psi}$, maximum water cut of 0.5 . The capacity of a separator is a total oil production rate of 165000 barrels per day, and total gas injection rate of 280 million cubic feet per day.

\subsubsection{BFGS and SR1 algorithms.}

In the optimization context, the Broyden- Fletcher- Goldfarb- Shanno $(B F G S)$ algorithm and symmetric rank one (SRI) is classified as quasi-Newton methods that are applied for solving nonlinear optimization problems iteratively [31-33]. The Newton's method requires the Jacobian and Hessian respectively for finding zeros, and extrema of a function. However, Quasi-Newton methods are based on Newton's method for solving problems that the Jacobian or Hessian is unavailable or are too expensive to compute at every iteration. In other words, it is not required to compute the Hessian matrix directly in quasiNewton methods, and it can be updated using successive gradient vectors calculations. One of the most important advantages of quasi-Newton methods over Newton's method is that the Hessian matrix does not need to be inverted, and consequently, the computational cost reduces. Quasi-Newton methods usually generate an estimate of hessian inverse directly. BFGS and SR1 algorithms might consist of following steps to reach to the solution from an initial guess $X_{0}$ and an approximate Hessian matrix $\mathrm{B}_{0}$ :

1. Select an initial guess, $X_{0}$, where $X_{0}$ is a vector in $R^{n}$, define approximation of the Hessian to the $N_{m} \times N_{m}$ identity matrix as a real-symmetric positive-definite $\left(B_{0}=I_{N m} \times N m\right)$ and set the iteration index $\mathrm{k}$ equal to zero.

2. Compute the objective function gradient, namely $\nabla f\left(X_{k}\right)$. The objective function is NPV that should be minimized. The gradient of $f(x)$ is calculated using the central finite difference method with the perturbation size of $(h)$ as follow:

$$
\nabla f\left(X_{k}\right)=\frac{f\left(X_{k}+h\right)-f\left(X_{k}-h\right)}{2 h}
$$

3. Compute the search direction of as follow:

$$
\Delta \mathrm{X}_{\mathrm{k}}=-\mathrm{B}_{\mathrm{k}}^{-1} \nabla \mathrm{f}\left(\mathrm{X}_{\mathrm{k}}\right)
$$

where $\mathrm{B}_{\mathrm{k}}^{-1}$ is the hessian inverse at iteration index $\mathrm{k}$.

4. Perform line search in the search direction to find the step size $\alpha_{\mathrm{k}} \geq 0$ in order to obtain the next point $\mathrm{X}_{k+1}$ by minimizing $\mathrm{f}\left(\mathrm{X}_{\mathrm{k}}+\alpha_{\mathrm{k}} \Delta \mathrm{X}_{\mathrm{k}}\right)$ over the scalar $\alpha_{\mathrm{k}}>0$.

5. Update the current solution as follow:

$$
\mathrm{X}_{\mathrm{k}+1}=\mathrm{X}_{\mathrm{k}}+\alpha_{\mathrm{k}} \Delta \mathrm{X}_{\mathrm{k}}
$$


6. Check for convergence. Convergence can be checked by observing the norm of the gradient $\left\|\nabla f\left(\mathrm{X}_{\mathrm{k}}\right)\right\|$. If the convergence criteria are satisfied, the approximation of the minimizing variables is set equal to $X_{k+1}$. If not, go to the next step.

7. Calculate the difference between two consecutive gradients as follow:

$$
\mathrm{y}_{\mathrm{k}}=\nabla \mathrm{f}\left(\mathrm{x}_{\mathrm{k}+1}\right)-\nabla \mathrm{f}\left(\mathrm{x}_{\mathrm{k}}\right)
$$

8. Update the Hessian matrix based on BFGS or SR1 as follow:

$$
\begin{aligned}
& \mathrm{B}_{\mathrm{k}+1}=\mathrm{B}_{\mathrm{k}}+\frac{\mathrm{y}_{\mathrm{k}} \mathrm{y}_{\mathrm{k}}^{\mathrm{T}}}{\mathrm{y}_{\mathrm{k}}^{\mathrm{T}} \Delta \mathrm{x}_{\mathrm{k}}}-\frac{\mathrm{B}_{\mathrm{k}} \Delta \mathrm{x}_{\mathrm{k}}\left(\mathrm{B}_{\mathrm{k}} \Delta \mathrm{x}_{\mathrm{k}}\right)^{\mathrm{T}}}{\Delta \mathrm{x}_{\mathrm{k}}^{\mathrm{T}} \mathrm{B}_{\mathrm{k}} \Delta \mathrm{x}_{\mathrm{k}}} \text { for BFGS } \\
& \mathrm{B}_{\mathrm{k}+1}=\mathrm{B}_{\mathrm{k}}+\frac{\left(\mathrm{y}_{\mathrm{k}}-\mathrm{B}_{\mathrm{k}} \Delta \mathrm{x}_{\mathrm{k}}\right)\left(\mathrm{y}_{\mathrm{k}}-\mathrm{B}_{\mathrm{k}} \Delta \mathrm{x}_{\mathrm{k}}\right)^{\mathrm{T}}}{\left(\mathrm{y}_{\mathrm{k}}-\mathrm{B}_{\mathrm{k}} \Delta \mathrm{x}_{\mathrm{k}}\right)^{\mathrm{T}} \Delta \mathrm{x}_{\mathrm{k}}} \text { for SR1 }
\end{aligned}
$$

9. Set the iteration index $\mathrm{k}$ equal to $\mathrm{k}+1$ and go to step 2 .

In BFGS and SR1 algorithm, If $\mathrm{B}_{0}$ is initialized with $\mathrm{B}_{0}=\mathrm{I} \mathrm{Nm} \times \mathrm{Nm}$, the first step will be equivalent to the gradient descent method, but as the algorithm progresses, an improved approximation of the Hessian at each iteration $\left(\mathrm{B}_{\mathrm{k}}\right)$ is generated. The gradient information of NPV with respect to individual good control rates (production and injection) is obtained using central difference based on Eq. 5 with perturbation size of 1 barrel per day for oil production rates, and 1000 cubic feet per day for gas injection rates during one year of optimization. The gradients are calculated using the simultaneous perturbation of all variables several times. Controls should be updated each year during a 17-year production plan. This leads to a constrained optimization problem with 374 decision variables that makes it a challenging task for a real and large scale full-field integrated model. This optimization problem is solved by applying BFGS and SR1.

\subsection{Discussion.}

This section summarizes the main findings from optimizing the production of a real mature oil field using BFGS and SR1. In this study, the optimal well controls were estimated for the seventeen years of the reservoir life using quasi-newton optimization algorithms based on the integrated reservoir model of the oil field. The optimization problem is to estimate the gas injection rates for injectors and oil production rates for producers, which maximize the NPV over the specified years of production.

To commence the optimization process, a feasible initial guess is required. In this regard, the initial guess for the rate controls of each gas injector is set equal to $52.5 \mathrm{MMscf} / \mathrm{D}$, and the initial guess for oil rate controls for each producer is equal to half of its corresponding observed maximum oil production rate based on the field production history. To calculate the NPV, it is required to set the oil sale price and various costs. The economic parameters and additional optimization settings are listed in Table 4. The wells and surface facilities constraints, including maximum oil production rate, gas injection rate, the capacity of a separator, and gas injection manifold, are listed in Table 5.

Table 4. Economic parameters and additional optimization settings.

\begin{tabular}{l|l} 
Properties & Value \\
\hline Oil revenue (\$/STB) & 100 \\
\hline Water production handling cost (\$/STB) & 5 \\
\hline Water injection cost $(\$ / \mathrm{STB})$ & 5 \\
\hline Gas injection cost $(\$ / 1000 \mathrm{SCF})$ & 2.3 \\
\hline Maximum oil production rate per well (STB/D) & 16000 \\
\hline Maximum gas injection rate per well (MMSCF/D) & 105
\end{tabular}


Table 5. Individual good controls and surface facility constraints.

\begin{tabular}{l|l|l|l} 
Name & Type & Maximum rate & Unit \\
\hline W1 & Producer & 5000 & Bbl/Day \\
\hline W2 & Producer & 13555 & Bbl/Day \\
\hline W3 & Producer & 10550 & Bbl/Day \\
\hline W4 & Producer & 5820 & Bbl/Day \\
\hline W5 & Producer & 550 & Bbl/Day \\
\hline W6 & Producer & 5010 & Bbl/Day \\
\hline W7 & Producer & 13300 & Bbl/Day \\
\hline W8 & Producer & 12340 & Bbl/Day \\
\hline W9 & Producer & 15550 & Bbl/Day \\
\hline W10 & Producer & 9500 & Bbl/Day \\
\hline W11 & Producer & 13300 & Bbl/Day \\
\hline W12 & Producer & 15300 & Bbl/Day \\
\hline W13 & Producer & 13700 & Bbl/Day \\
\hline W15 & Producer & 10800 & Bbl/Day \\
\hline W16 & Producer & 12500 & Bbl/Day \\
\hline W17 & Producer & 5300 & Bbl/Day \\
\hline W18 & Producer & 7400 & Bbl/Day \\
\hline W19 & Producer & 1620 & Bbl/Day \\
\hline W20 & Producer & 11000 & Bbl/Day \\
\hline W21 & Injector & 105000 & MScf/Day \\
\hline W22 & Injector & 105000 & MScf/Day \\
\hline Separator & Injector & 105000 & MScf/Day \\
\hline Separator & Oil & 165000 & Bbl/Day \\
\hline Injection manifold & Gas & 280000 & MScf/Day \\
\hline & Gas & 280000 & MScf/Day
\end{tabular}

The optimization was done using BFGS and SR1 algorithms. Figure 2 shows the performance of the two quasi-newton methods over the current production plan provided by the oil industry for 17 years of production and injection based on the original integrated model. Figure 2 shows that BFGS is superior over the SR1 for the long term optimization.

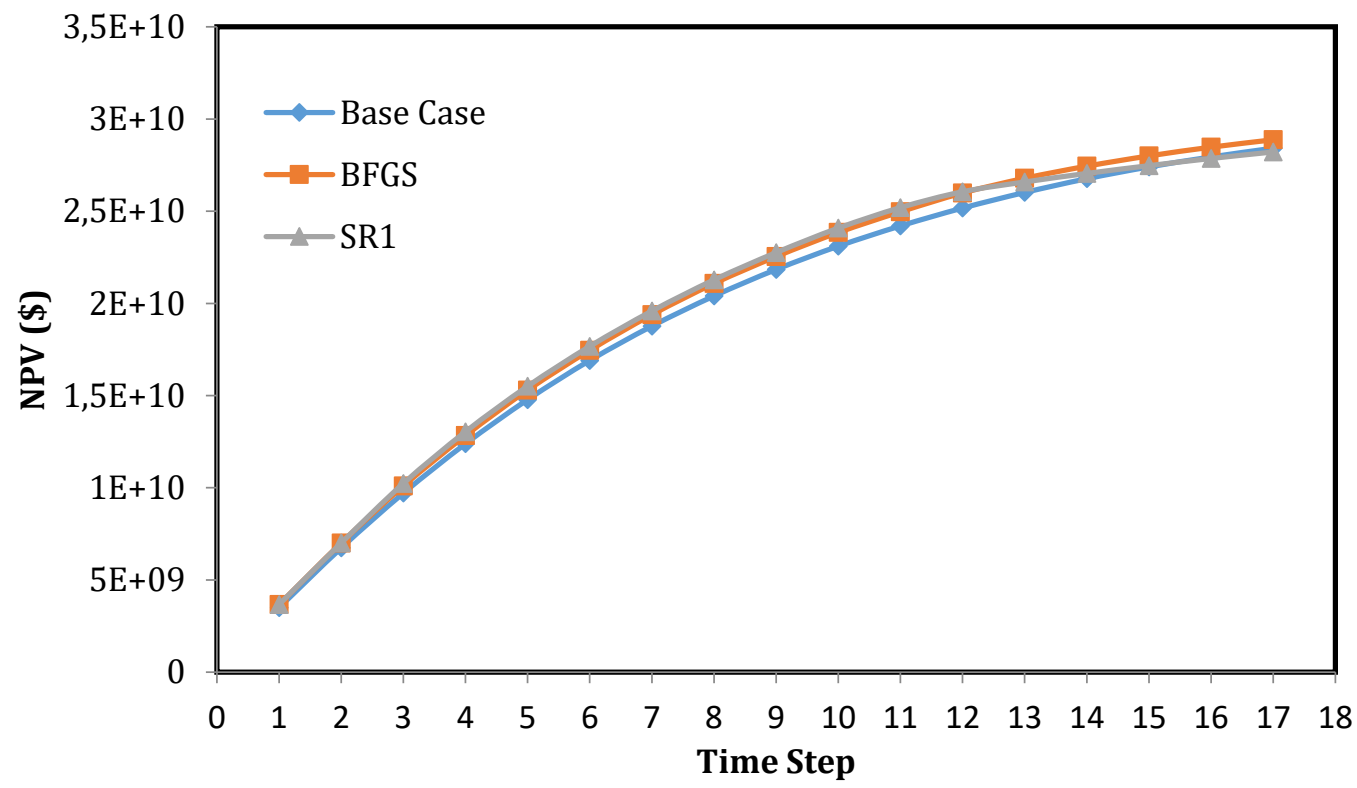

Figure 2. Comparison of optimized NPV using BFGS and SR1 over the NPV of current production and injection plan provided by the oil industry.

In order to compare the performance of BFGS and SR1 over the current production and injection plan, the optimized NPV of BFGS and SR1 methods are given in Table 6. For the first 12 time steps, the SR1 performance is better than BFGS because the cumulative oil production is higher than that of the BFGS. However, as the production time increases over time step 12, the performance of the SR1 reduces below that of the BFGS. For the last 2 years 
of production optimization, the optimized NPV using SR1 is even much lower than the NPV of current production and injection plan because SR1 presents production and injection rates that increase water production and its handling costs. In addition, performing optimization using SR1 results in excessive gas production, which reduces the reservoir energy to produce oil, and simultaneously increases gas injection costs. Table 7 summarizes the optimized production and injection rates for all wells based on BFGS algorithm.

Table 6. the optimization results using BFGS and SR1 over the NPV of the base case model.

\begin{tabular}{|c|c|c|c|c|c|}
\hline $\begin{array}{l}\text { Time } \\
\text { Step } \\
\#\end{array}$ & Base case NPV (\$) & SR1 NPV (\$) & BFGS NPV (\$) & $\begin{array}{ll}\text { SR1 } & \text { NPV } \\
\text { improvement } & \text { over } \\
\text { Base Case NPV } & \end{array}$ & $\begin{array}{ll}\text { BFGS } & \text { NPV } \\
\text { improvement } & \text { over } \\
\text { Base Case NPV } & \end{array}$ \\
\hline 1 & $3.496 \mathrm{E}+09$ & $3.670 \mathrm{E}+09$ & $3.668 \mathrm{E}+09$ & 4.976 & 4.920 \\
\hline 2 & $6.736 \mathrm{E}+09$ & $7.007 \mathrm{E}+09$ & $7.004 \mathrm{E}+09$ & 4.018 & 3.972 \\
\hline 3 & $9.707 \mathrm{E}+09$ & $1.023 \mathrm{E}+10$ & $1.009 \mathrm{E}+10$ & 5.394 & 3.976 \\
\hline 4 & $1.239 \mathrm{E}+10$ & $1.304 \mathrm{E}+10$ & $1.283 \mathrm{E}+10$ & 5.269 & 3.578 \\
\hline 5 & $1.478 \mathrm{E}+10$ & $1.551 \mathrm{E}+10$ & $1.530 \mathrm{E}+10$ & 4.917 & 3.497 \\
\hline 6 & $1.690 \mathrm{E}+10$ & $1.767 \mathrm{E}+10$ & $1.746 \mathrm{E}+10$ & 4.536 & 3.261 \\
\hline 7 & $1.877 \mathrm{E}+10$ & $1.959 \mathrm{E}+10$ & $1.939 \mathrm{E}+10$ & 4.362 & 3.300 \\
\hline 8 & $2.040 \mathrm{E}+10$ & $2.128 \mathrm{E}+10$ & $2.108 \mathrm{E}+10$ & 4.296 & 3.322 \\
\hline 9 & $2.184 \mathrm{E}+10$ & $2.276 \mathrm{E}+10$ & $2.254 \mathrm{E}+10$ & 4.201 & 3.209 \\
\hline 10 & $2.310 \mathrm{E}+10$ & $2.408 \mathrm{E}+10$ & $2.384 \mathrm{E}+10$ & 4.253 & 3.210 \\
\hline 11 & $2.420 \mathrm{E}+10$ & $2.520 \mathrm{E}+10$ & $2.497 \mathrm{E}+10$ & 4.112 & 3.163 \\
\hline 12 & $2.517 \mathrm{E}+10$ & $2.606 \mathrm{E}+10$ & $2.599 \mathrm{E}+10$ & 3.523 & 3.250 \\
\hline 13 & $2.602 \mathrm{E}+10$ & $2.658 \mathrm{E}+10$ & $2.679 \mathrm{E}+10$ & 2.189 & 2.963 \\
\hline 14 & $2.676 \mathrm{E}+10$ & $2.705 \mathrm{E}+10$ & $2.744 \mathrm{E}+10$ & 1.068 & 2.537 \\
\hline 15 & $2.740 \mathrm{E}+10$ & $2.747 \mathrm{E}+10$ & $2.800 \mathrm{E}+10$ & 0.253 & 2.179 \\
\hline 16 & $2.794 \mathrm{E}+10$ & $2.786 \mathrm{E}+10$ & $2.847 \mathrm{E}+10$ & -0.296 & 1.893 \\
\hline 17 & $2.842 \mathrm{E}+10$ & $2.821 \mathrm{E}+10$ & $2.888 \mathrm{E}+10$ & -0.721 & 1.605 \\
\hline
\end{tabular}

Table 7. The optimized individual well controls using BFGS from $\mathrm{TS}=1$ to $\mathrm{TS}=10$.

\begin{tabular}{|c|c|c|c|c|c|c|c|c|c|c|c|c|c|c|c|c|c|}
\hline Well \# & TS1 & TS2 & TS3 & TS4 & TS5 & TS6 & TS7 & TS8 & TS9 & TS10 & TS11 & TS12 & TS13 & TS14 & TS15 & TS16 & TS17 \\
\hline W1 & 4774 & 4774 & 4774 & 837 & 1 & 5000 & 1 & 1 & 5000 & 3754 & 5000 & 4778 & 213 & 313 & 2148 & 1519 & 1 \\
\hline W2 & 9634 & 13555 & 13555 & 8584 & 1 & 13555 & 10074 & 13555 & 13555 & 8414 & 1 & 7 & 10495 & 65 & 12550 & 6006 & 1013 \\
\hline W3 & 10058 & 10550 & 9756 & 10521 & 10550 & 10550 & 10550 & 10550 & 10550 & 10550 & 10550 & 10550 & 2625 & 10029 & 4853 & 5242 & 6071 \\
\hline $\mathrm{W} 4$ & 2562 & 2905 & 1 & 1 & 5820 & 5820 & 5820 & 1 & 5820 & 5820 & 5820 & 5441 & 1 & 3931 & 1 & 2691 & 4184 \\
\hline W5 & 1262 & 1 & 1 & 227 & 1 & 550 & 1 & 1 & 550 & 550 & 1 & 1 & 1 & 1 & 23 & 1 & 1 \\
\hline W6 & 5148 & 2151 & 5010 & 3578 & 5010 & 5010 & 5010 & 5010 & 5010 & 5010 & 1 & 223 & 50 & 4402 & 2527 & 2539 & 1854 \\
\hline W7 & 7459 & 2471 & 11046 & 13300 & 13300 & 13300 & 13300 & 13300 & 13300 & 12341 & 13300 & 13300 & 12248 & 7960 & 778 & 6031 & 1157 \\
\hline W8 & 12949 & 10700 & 9178 & 9833 & 12340 & 12340 & 12340 & 12340 & 2494 & 8524 & 12340 & 11902 & 10556 & 1816 & 8275 & 5965 & 2675 \\
\hline W9 & 2031 & 7693 & 15550 & 15215 & 1 & 1 & 1 & 15550 & 1 & 2574 & 15550 & 15237 & 9290 & 1341 & 6482 & 7793 & 6587 \\
\hline W10 & 8231 & 4033 & 4822 & 6440 & 9500 & 1 & 1 & 9500 & 1 & 6904 & 9500 & 9153 & 1 & 5099 & 7836 & 4339 & 1 \\
\hline W11 & 11437 & 13300 & 10661 & 12679 & 13300 & 1 & 13300 & 13300 & 13300 & 8588 & 13300 & 13300 & 13214 & 2388 & 2664 & 7661 & 11262 \\
\hline W12 & 7238 & 10802 & 2038 & 892 & 15300 & 15300 & 15300 & 15300 & 15300 & 6593 & 15300 & 14953 & 15300 & 3113 & 3847 & 8319 & 11954 \\
\hline W13 & 10426 & 6797 & 13700 & 13317 & 9147 & 13700 & 13700 & 2809 & 13700 & 13443 & 13700 & 13328 & 13392 & 8475 & 4239 & 7208 & 9766 \\
\hline W14 & 5882 & 2884 & 10800 & 4934 & 1 & 10800 & 10800 & 10800 & 10800 & 6037 & 10800 & 10800 & 1653 & 6852 & 5381 & 5439 & 5251 \\
\hline W15 & 9666 & 3314 & 3098 & 1 & 12500 & 1 & 12500 & 1 & 1 & 7683 & 12500 & 12121 & 1471 & 10215 & 10902 & 6229 & 9450 \\
\hline W16 & 3721 & 5300 & 5300 & 4247 & 5300 & 1 & 1 & 5300 & 1 & 3593 & 5300 & 5300 & 4037 & 843 & 5086 & 2318 & 1 \\
\hline W17 & 4768 & 7400 & 1194 & 7400 & 1 & 1 & 7400 & 7400 & 7400 & 7400 & 7400 & 7396 & 6045 & 4697 & 5685 & 4275 & 7400 \\
\hline W18 & 1 & 1620 & 1 & 1198 & 1620 & 1620 & 1620 & 1620 & 1620 & 1163 & 1620 & 1339 & 1620 & 580 & 1 & 1620 & 1601 \\
\hline W19 & 10340 & 5174 & 11000 & 11000 & 11000 & 11000 & 1 & 11000 & 11000 & 7544 & 11000 & 10628 & 663 & 3016 & 9432 & 5927 & 5764 \\
\hline W20 & 103162 & 55611 & 58687 & 54239 & 1 & 1 & 105000 & 1 & 1 & 94080 & 105000 & 104562 & 40901 & 46436 & 29786 & 52673 & 55849 \\
\hline W21 & 100251 & 53468 & 55264 & 51984 & 105000 & 105000 & 1 & 105000 & 105000 & 10963 & 1 & 1 & 104237 & 62740 & 33417 & 52293 & 48049 \\
\hline W22 & 28512 & 53928 & 49807 & 44453 & 105000 & 1 & 105000 & 1 & 105000 & 99577 & 105000 & 104996 & 103523 & 26628 & 31589 & 51919 & 46487 \\
\hline
\end{tabular}

Figure 3 shows the NPV improvement over the base case NPV for all time steps using BFGS and SR1. As can be seen, for the first 2 time steps, the BFGS and SR1 have the same performance. For the first 2 time steps, performing optimization averagely improves the NPV over the base case NPV, respectively, by $4.5 \%$ and $4.47 \%$ using SR1 and BFGS. From time step 3 to 12, SR1 outperforms BFGS. The NPV improves by $4.49 \%$ and $3.38 \%$ using SR1 and BFGS, respectively over the base case NPV. For the last 5 time steps from 13 to 17, BFGS outperforms SR1. The NPV improves by $2.39 \%$ using BFGS over the base case. As can be seen clearly from Figure 3 for the last 2 time steps, the performance of SR1 reduces sharply even below the NPV of the current production and injection plan as a result of excessive water and gas production rates. In this case, SR 1 reduces the NPV by $0.51 \%$ over the base case NPV. 


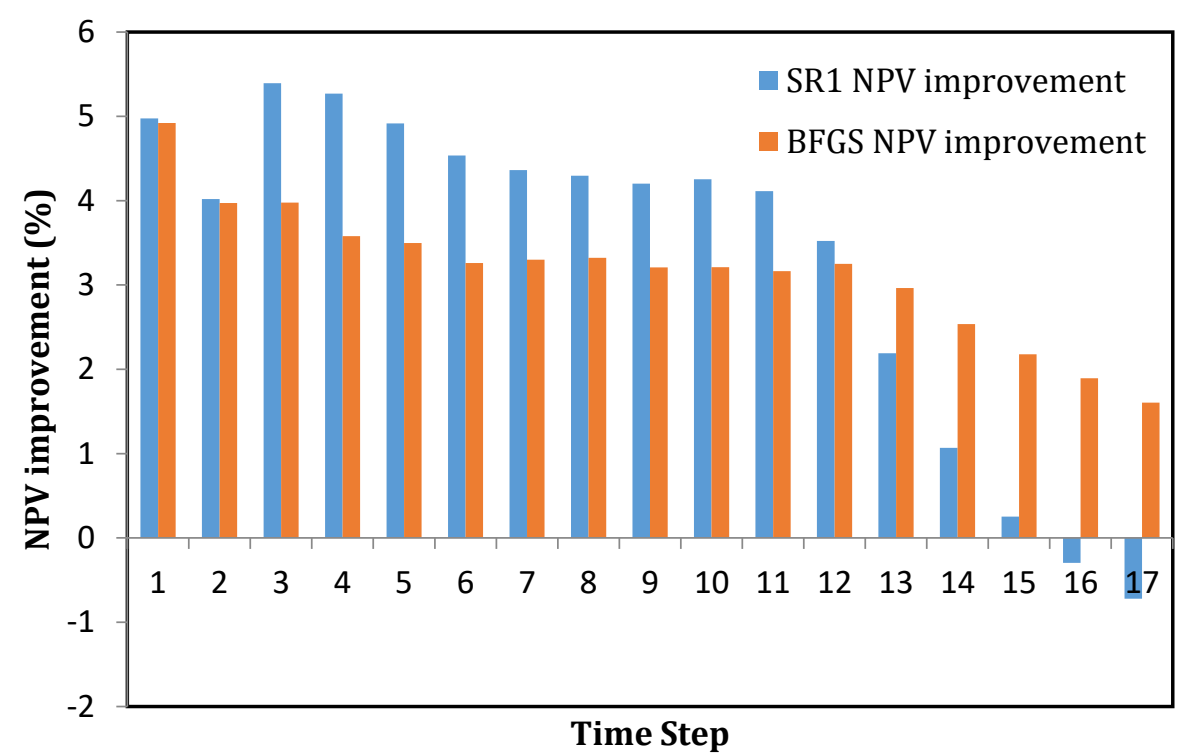

Figure 3. NPV improvement over the NPV of the base case model for all time steps using BFGS and SR1. For the first 12 time steps, SR1 outperforms BFGS, and for the last 5 time steps, BFGS is superior over SR1. For the last 2 time steps, the NPV provided using SR1 reduces below the NPV of the base case model.

In sum, the production optimization from the real oil field using BFGS and SR1 reveals that the current production and injection plan provided by the oil industry is suboptimal, and it could be improved by applying suitable optimization algorithms. The optimization process using BFGS and SR1 shows that the BFGS method is ultimately superior over the SR1 method for long term optimization of 17 years of production and injection. However, it is most favorable to use SR1 algorithm for short time scale optimization in order to maximize the return from the field. Finally, the combination of BFGS and SR1 could give a much better production and injection plan over the individual optimization algorithms in maximizing the NPV for the long term production optimization.

This section may be divided by subheadings. It should provide a concise and precise description of the experimental results, their interpretation as well as the experimental conclusions that can be drawn.

\section{Conclusions}

In the present study, a framework based on the quasi-newton algorithms was proposed to maximize the NPV in life-cycle production optimization. Two quasi-newton methods based on BFGS and SR1 were used. A full-scale mature oil field located in the southwest of Iran was provided to demonstrate the robustness of the proposed framework. Through the numerical simulations and performing optimization, it can be observed that the proposed framework can be effectively used to maximize the returns from the field. The performance of the BFGS algorithm was compared with that of the SR1 algorithm over the current production and injection plan provided by the oil industry.

It was observed that the production optimization using BFGS is superior over the SR1 in maximizing NPV in the 17 years of production and injection. Optimizing production from the field using BFGS algorithm results in lower handling costs in terms of produced water and gas injection. On the other hand, the performance of the SR1 first increases over the base case for 15 years and then decreases over the base case for the last 2 years of production as a result of increased water and gas production. Although for the first 12 years of production and 
injection, the performance of the SR1 method is much better than BFGS as a result of increased cumulative oil production, optimization using SR1 over the 17 years of production reduces the NPV by $0.51 \%$ lower than that of the current plan. This indicates that the optimized production and injection rates using SR1 eventually results in increased cumulative water and excessive gas production with respect to BFGS. Therefore, it is very important to characterize the time scale of production optimization in order to reach a reliable production plan which maximizes NPV. Results show that the production optimization using BFGS increases the NPV from $1.605 \%$ to $4.920 \%$ for various time steps over the current scheduled production and injection plan provided by the oil industry.

\section{Funding}

This research was funded by Iran National Science Foundation (INSF) Grant\#), grant number 96004608, Tehran, Iran.

\section{Acknowledgments}

The authors fully acknowledge the financial support from Iran National Science Foundation, Tehran, Iran.

\section{Conflicts of Interest}

The authors declare no conflict of interest.

\section{References}

1. Yeten, B.; Durlofsky, L.; Aziz, K. Optimization of nonconventional well type, location, and trajectory. SPE 86880-PA 2003, 8, https://doi.org/10.2118/86880-PA.

2. Güyagüler, B.; Horne, R.N. Uncertainty assessment of well-placement optimization. SPE 87663-PA 2004, 7, https://doi.org/10.2118/87663-PA.

3. Zandvliet, M.; Handels, M.; Essen, G.V.; Brouwer, R.; Jansen, J.D. Adjoint-based well-placement optimization Under production constraints. SPE105797-PA 2008, 13, https://doi.org/10.2118/105797-PA.

4. Hazlett, R.D.; Babu, D.K. Optimal well placement in heterogeneous reservoirs via semi-analytic modeling. SPE 84281-PA 2005, 10, https://doi.org/10.2118/84281-PA.

5. Onwunalu, J.E.; Durlofsky, L. A new well-Pattern-optimization procedure for large-scale field development. SPE 124364-PA 2011, 16, https://doi.org/10.2118/124364-PA.

6. Wang, H.; Echeverría-Ciaurri, D.; Cominelli, A. Optimal well placement Under uncertainty using a retrospective optimization framework. SPE141950-PA 2012, 17, https://doi.org/10.2118/141950-PA.

7. Bouzarkouna, Z.; Didier, Y. Ding. Partially separated meta models With evolution strategies for wellplacement optimization. SPE-143292-PA 2013, 18, https://doi.org/10.2118/143292-PA.

8. Isebor, O.J.; EcheverríaCiaurri, D.; Durlofsky, L. Generalized field-development optimization With derivative-free procedures. SPE-163631-PA 2014, 19, https://doi.org/10.2118/163631-PA.

9. Rasouli, H.; Rashidi, F.; Karimi, B.; Khamehchi, E. A surrogate integrated production modeling approach to long-term gas-lift allocation optimization. Chem. Eng. Commun 2015, 202, 647-654, https://doi.org/10.1080/00986445.2013.863186.

10. Naderi, M.; Khamehchi, E. Nonlinear risk optimization approach to water drive gas reservoir production optimization using DOE and artificial intelligence. J. Nat. Gas. Sci. Eng 2016, 31, 575-584, https://doi.org/10.1016/j.jngse.2016.03.069.

11. Foroud, T.; Seifi, A.; AminShahidy, B. An efficient optimization process for hydrocarbon production in presence of geological uncertainty using a clustering method: A case study on Brugge field. Journal of Natural Gas Science and Engineering 2016, 32, 476-490, https://doi.org/10.1016/j.jngse.2016.04.059.

12. Foroud, T.; Seifi, A.; AminShahidy, B. An efficient optimization process for hydrocarbon production in presence of geological uncertainty using a clustering method: A case study on Brugge field. Journal of Natural Gas Science and Engineering 2016, 32, 476-490, https://doi.org/10.1016/j.petrol.2016.12.028.

13. Naderi, M.; Khamehchi, E. Application of DOE and metaheuristic bat algorithm for well placement and individual well controls optimization. Journal of Natural Gas Science and Engineering 2017, 46, 47-58, https://doi.org/10.1016/j.jngse.2017.07.012. 
14. Chen, B.; Fonseca, R.-M.; Leeuwenburgh, O.; Reynolds, A.C. Minimizing the Risk in the robust life-cycle production optimization using stochastic simplex approximate gradient. Journal of Petroleum Science and Engineering 2017, 153, 331-344, https://doi.org/10.1016/j.petrol.2017.04.001.

15. Fonseca, R.M.; Kahrobaei, S.S.; van Gastel L.J.T.; Leeuwenburgh O.; and Jansen J.D. 2015. Quantification of the impact of ensemble size on the quality of an ensemble gradient using principles of hypothesis testing, SPE 173236-PA 2015, https://doi.org/10.2118/173256-PA.

16. Fonseca, R.R.-M.; Chen, B.; Jansen, J.D.; Reynolds, A. A Stochastic Simplex Approximate Gradient (StoSAG) for optimization under uncertainty. International Journal for Numerical Methods in Engineering 2017, 109, 1756-1776, https://doi.org/10.1002/nme.5342.

17. Ezzati S.; Khamehchi E. Sandstone Reservoir Wettability Alteration Due to Water Softening: Impact of Silica Nanoparticles on Sand Production Mechanism. Biointerface Research in Applied Chemistry 2020, 10, 6328-6342, https://doi.org/10.33263/BRIAC105.63286342.

18. Ejtemaee P.; Khamehchi E. Experimental investigation of rheological properties and formation damage of water-based drilling fluids in the presence of $\mathrm{Al} 2 \mathrm{O} 3, \mathrm{Fe} 3 \mathrm{O} 4$, and $\mathrm{TiO} 2$ nanoparticles. Biointerface Research in Applied Chemistry 2020, 10, 5886 - 5894, https://doi.org/10.33263/BRIAC104.886894.

19. Bahri A.; Khamehchi E. Interfacial Phenomena Effect on Sand Production Due to Optimized Smart Water with/without the Presence of Nanoparticles. Biointerface Research in Applied Chemistry 2020, 10, 66526668.

20. Kim, J.; Yang, H.; Choe, J. Robust optimization of the locations and types of multiple wells using CNN based proxy models. Journal of Petroleum Science and Engineering 2020, 193, https://doi.org/10.1016/j.petrol.2020.107424.

21. Zhang, L.; Li, Z.; Lai, F.; Li, H.; Adenutsi, C.D.; Wang, K.; Yang, S.; Xu, W. Integrated optimization design for horizontal well placement and fracturing in tight oil reservoirs. Journal of Petroleum Science and Engineering 2019, 178, 82-96, https://doi.org/10.1016/j.petrol.2019.03.006.

22. Liu, Z.; Forouzanfar, F.; Zhao, Y. Comparison of SQP and AL algorithms for deterministic constrained production optimization of hydrocarbon reservoirs. Journal of Petroleum Science and Engineering 2018, 171, 542-557, https://doi.org/10.1016/j.petrol.2018.06.063.

23. Kim, J.; Kang, B.; Jeong, H.; Choe, J. Field development optimization using a cooperative micro-particle swarm optimization with parameter integration schemes. Journal of Petroleum Science and Engineering 2019, 183, https://doi.org/10.1016/j.petrol.2019.106416.

24. Zhang, H.; Zhang, K.; Zhang, L.; Sheng, J.; Yao, J.; Wang, J.; Yang, Y. Construction and optimization of adaptive well pattern based on reservoir anisotropy and uncertainty. Journal of Petroleum Science and Engineering 2019, 181, https://doi.org/10.1016/j.petrol.2019.106252.

25. Jesmani, M.; Jafarpour, B.; Bellout, M.C.; Foss, B. A reduced random sampling strategy for fast robust well placement optimization. Journal of Petroleum Science and Engineering 2020, 184, https://doi.org/10.1016/j.petrol.2019.106414.

26. Janiga, D.; Czarnota, R.; Stopa, J.; Wojnarowski, P. Self-adapt reservoir clusterization method to enhance robustness of well placement optimization. Journal of Petroleum Science and Engineering 2019, 173, 3752, https://doi.org/10.1016/j.petrol.2018.10.005.

27. Khan, R.A.; Awotunde, A.A. determination of vertical/horizontal well type from generalized field development optimization. Journal of Petroleum Science and Engineering 2018, 162, 652-665, https://doi.org/10.1016/j.petrol.2017.10.083.

28. Dang, C.; Nghiem, L.; Nguyen, N.; Yang, C.; Chen, Z.; Bae, W. Modeling and optimization of alkalinesurfactant-polymer flooding and hybrid enhanced oil recovery processes. Journal of Petroleum Science and Engineering 2018, 169, 578-601, https://doi.org/10.1016/j.petrol.2018.06.017.

29. Song, X.; Liu, Y.; Xue, L.; Wang, J.; Zhang, J.; Wang, J.; Jiang, L.; Cheng, Z. Time-series well performance prediction based on Long Short-Term Memory (LSTM) neural network model. Journal of Petroleum Science and Engineering 2020, 186, https://doi.org/10.1016/j.petrol.2019.106682.

30. Wang, S.; Chen, Z.; Chen, S. Applicability of deep neural networks on production forecasting in Bakken shale reservoirs. Journal of Petroleum Science and Engineering 2019, 179, 112-125, https://doi.org/10.1016/j.petrol.2019.04.016.

31. Fletcher, R. Practical methods of optimization. 2nd ed, John Wiley \& Sons: New York, USA, 2000.

32. Conn, A.R.; Gould, N.I.M.; Toint, P.L. Convergence of quasi-Newton matrices generated by the symmetric rank one update. Mathematical Programming 1991, 50, 177-195, https://doi.org/10.1007\%2FBF01594934.

33. Byrd, R.H.; Khalfan, H.F.; Schnabel, R.B. Analysis of a Symmetric Rank-One Trust Region Method. SIAM Journal on Optimization 1996, 6, 1025-1039, https://doi.org/10.1137/S1052623493252985. 